\title{
Coronary plaque tissue characterization in patients with premature coronary artery disease
}

\author{
Jianchang Xie ${ }^{1}$. Jie $\mathrm{Qi}^{2} \cdot$ Hengyi Mao ${ }^{3} \cdot$ Ningfu Wang $^{1} \cdot$ Xianhua Ye $^{1} \cdot$ Liang Zhou $^{1} \cdot$ Guoxin Tong ${ }^{1}$ Jianmin Yang ${ }^{1}$. \\ Hao Pan ${ }^{1}$. Jinyu Huang ${ }^{1}$
}

Received: 16 July 2019 / Accepted: 11 February 2020 / Published online: 20 February 2020

(C) The Author(s) 2020

\begin{abstract}
Premature coronary artery disease (CAD) studies rarely involve coronary plaque characterization. We characterize coronary plaque tissue by radiofrequency intravascular ultrasound (IVUS) in patients with premature CAD. From July 2015 to December 2017, 220 patients from the Department of Cardiology, Affiliated Hangzhou First People's Hospital, Zhejiang University School of Medicine with first occurrence of angina or myocardial infarction within 3 months were enrolled. Patients with premature CAD $(n=47$, males aged $<55$ years, and females aged $<65$ years) or later CAD $(n=155)$ were retrospectively compared for cardiovascular risk factors, laboratory examination findings, coronary angiography data, gray-scale IVUS, and iMap-IVUS. The mean age was $53.53 \pm 7.24$ vs. $70.48 \pm 8.74$ years $(\mathrm{p}<0.001)$. The groups were similar for traditional coronary risk factors except homocysteine $(18.60 \pm 5.15$ vs. $17.08 \pm 4.27 \mu \mathrm{mol} / \mathrm{L}, \mathrm{p}=0.043)$. After matching for baseline characteristics, LDL cholesterol (LDL-C) was higher for premature CAD than later CAD ( $2.50 \pm 0.96 \mathrm{vs.} 2.17 \pm 0.80 \mathrm{mmol} / \mathrm{L}$, $\mathrm{p}=0.019)$. Before the matching procedure, the premature CAD group had shorter target lesion length [18.50 (12.60-32.00) vs. $27.90(18.70-37.40) \mathrm{mm}, \mathrm{p}=0.002]$, less plaque volume [175.59 (96.60-240.50) vs. $214.73(139.74-330.00) \mathrm{mm}^{3}$, $\mathrm{p}=0.013]$ than the later CAD group. After the matching procedure, the premature CAD group appeared to be less plaque burden $(72.69 \pm 9.99$ vs. $74.85 \pm 9.80 \%, p=0.005)$, and positive remodeling $(1.03 \pm 0.12$ vs. $0.94 \pm 0.18, p=0.034)$, and lower high risk feature incidence $(\mathrm{p}=0.006)$ than the later $\mathrm{CAD}$ group. At the plaque's minimum lumen, premature CAD had more fibrotic $(\mathrm{p}<0.001)$, less necrotic $(\mathrm{p}=0.001)$ and less calcified areas $(\mathrm{p}=0.012)$. Coronary plaque tissue was more fibrotic with less necrotic and calcified components in premature than in later $\mathrm{CAD}$, and the range and degree of atherosclerosis were significantly lower.
\end{abstract}

Keywords Premature coronary heart disease $\cdot$ iMap intravascular ultrasound $\cdot$ Cardiac catheterization $\cdot$ Plaque

\section{Introduction}

Coronary artery disease (CAD) is the leading cause of mortality and much is known about the causes and risk factors for the disease [1]. CAD is a progressive disease that takes

Jinyu Huang

hjyuo@163.com

1 Department of Cardiology, Affiliated Hangzhou First People's Hospital, Zhejiang University School of Medicine, 261\# Huansha Road, Shangcheng District, Hangzhou 310006, China

2 Department of Cardiology, Wenzhou People's Hospital, Wenzhou, China

3 Department of Cardiology, Ningbo First Hospital, Ningbo, China time to develop [2]. Therefore, age is a significant contributory factor for CAD [3]. When CAD occurs in younger than expected patients it is considered to be premature CAD. However, the definition of premature CAD is difficult because of the variation in risk factors in different populations [4]. Chinese studies use the definition of men with onset age $<55$ years, and women with onset age $<65$ years [5]. The risk of developing CAD at a younger age appears to occur more often in Asian populations and particularly in South Asians [6], and is related to the established risk factors including thrombotic (smoking, low fruit/vegetable intake, fibrinogen, homocysteine) and atherosclerotic (hypertension, high fat diet, dyslipidemia) factors in combination with a genetic basis [7]. In China, despite understanding risk factors, the incidence of young CHD, in people $\leq 45$ years, is increasing [5]. 
Prior studies have examined the relationship between a family history of premature CAD and coronary artery calcium [8], plaque burden [9] in healthy relatives from families with premature CAD by computed tomography angiography (CTA). Coronary angiography is a well-established diagnostic modality for percutaneous coronary intervention (PCI) guidance, but more recent intravascular imaging techniques enhance the efficacy of lesion evaluation [10]. For example, advanced imaging has shown that the necrotic tissue volume is a potent predictor of periprocedural myocardial infarction after PCI [11]. Importantly, the incidence of an acute coronary event is determined by the extent and severity of the luminal stenosis, especially the characteristics of the plaque [12]. intravascular ultrasound (IVUS) has been developed to access plaque composition and define atherosclerotic lesion phenotype [13]. Classical greyscale IVUS has now been expanded with spectral analysis of back scattered radio frequency (RF) data. Based on pattern recognition of the RF signals, iMap-IVUS (Boston Scientific, Marlborough, MA) can provide quantitative analysis of plaque composition and classify them to four tissue types (fibrotic, lipidic, necrotic, and calcified) in vivo $[14,15]$.

While most studies on premature CAD focus on genetics and epidemiology, there are few studies related to coronary plaque characterization. In consideration of the genetic basis of premature $\mathrm{CAD}$, whether patients with premature $\mathrm{CAD}$ exhibit differences in plaque characteristics is currently not known. Therefore, an investigation into the plaque characteristics of premature CAD and how they compare with typical CAD might reveal important information on the development of this disease.

This study aimed to analyze the morphological, tissue, and phenotypical characteristics of atherosclerotic plaques using iMap-IVUS in patients with premature CAD and compare the differences with patients with CAD who developed the disease later.

\section{Methods}

\section{Study patients and materials}

In this single center study, from July 2015 to December 2017, a total of 220 patients from the Department of Cardiology, Affiliated Hangzhou First People's Hospital, Zhejiang University School of Medicine with first occurrence of angina or MI in 3 months were enrolled. These were consecutive patients who underwent diagnostic coronary angiography or PCI and were diagnosed with acute coronary syndrome (ACS) or stable CAD.

The inclusion criteria were as follows: (1) First occurrence of angina or myocardial infarction (MI) in 3 months. (2) Diagnosed with ACS or stable CAD by coronary angiography (CAG). The exclusion criteria were as follows: (1) Cholesterol-lowering therapy more than 1 month. (2) CAG showed in-stent restenosis and vision thrombus. (3) Inadequate imaging quality.

The patients were grouped into the premature CAD group and later CAD groups according to their age at diagnosis. Premature CAD was defined as patients aged less than 55 years in men and less than 65 years in women [5]. The older patients were defined as the later CAD group.

\section{Intravascular ultrasound}

The IVUS data were acquired with an iLab System and mechanical $40 \mathrm{MHz}$ IVUS catheter (Both Boston Scientific, Natick, MA, USA). The catheter was advanced beyond the target lesion, and imaging was performed during automatic pullback at a speed of $0.5 \mathrm{~mm} / \mathrm{s}$. RF signals were captured on every 4th IVUS frame for tissue compositional data. All recordings were assigned to randomly generated examination ID numbers corresponding to a list and archived to CDROM for later offline analysis. Based on the criteria of the American College of Cardiology Clinical Expert Consensus Document on IVUS [16], two experienced analysts who were unaware of the angiographic findings or the baseline clinical and lesion characteristics analyzed the gray-scale IVUS images. QIvus (iMap Basic Viewer 3.0, Medis medical imaging systems bv, Leiden, the Netherlands) was used for analysis.

In ACS group,culprit lesion was identified as target lesion. In stable CAD group, target lesion was defined as the lesion with the most severe plaque burden or needed be intervened firstly in multivessel CAD. In each target lesion, a cross sectional area (CSA) for the lumen and the external elastic membrane (EEM) was traced manually. Plaque burden was calculated as the ratio between plaque CSA (EEM minus lumen CSA) and EEM. Lumen and vessel volumes were calculated as the summation of lumen and EEM area in each measured image respectively. Total atheroma volume (TAV) was calculated as vessel volume - lumen volume. Percent atheroma volume (PAV) was calculated as (TAV/ vessel volume) $\times 100 \%$.

iMap software-classified plaque into four tissue components and produced color images (green for fibrous plaque, yellow for lipidic plaque, pink for necrotic plaque and blue for calcified plaque). A plaque that was unsuitable for analysis was defined as an acoustic shadowing area and volume behind calcification or wire artifact [17].

\section{Statistical analysis}

Categorical variables are presented as percentage frequencies and were analyzed using $\chi^{2}$ tests or Fisher exact tests as appropriate. Continuous variables are expressed as 
mean \pm standard deviation $(\mathrm{SD})$ or median (IQR) and were compared using the student's two-tailed unpaired $t$ test or Mann-Whitney test.

We performed a matching procedure to account for differences in baseline characteristics between premature CAD and later CAD. Every patient with premature CAD was matched to the one with later CAD by gender, body mass index (BMI), indication for catheterization (ACS or stable CAD), history of hypertension, diabetes mellitus, statin use, and current smoking.

Data analysis was performed with the Statistical Package for Social Sciences (SPSS) software on Windows version 24.0 (IBM corp. Armonk, NY, USA). All statistical tests were two-tailed and $\mathrm{p}$-values $<0.050$ were considered statistically significant.

\section{Results}

\section{Patients baseline characteristics}

From the 220 patients selected for inclusion in the study, 18 patients were excluded for in-stent restenosis, vision thrombus with angiographically, and an inadequate imaging quality. Finally, 202 patients were analyzed.

The baseline clinical characteristics of the total patient population are presented in Table 1 . There were 47 patients in the premature CAD group and 155 patients in later $\mathrm{CAD}$ group. The mean age was $53.53 \pm 7.24$ vs. $70.48 \pm 8.74$ years respectively $(\mathrm{p}<0.001)$. There were no significant differences between the two groups in terms of traditional coronary risk factors except homocysteine $(18.60 \pm 5.15 \mathrm{vs}$. $17.08 \pm 4.27 \mu \mathrm{mol} / \mathrm{L}, \mathrm{p}=0.043)$. Due to the different diagnostic criteria of premature CAD for different genders, the males in the premature CAD group was significantly lower than in the later CAD group (51.06 vs. $70.97 \%, p=0.014$ ). After the matching procedure, baseline clinical and procedural characteristics were similarly distributed between the two groups. The level of low-density lipoprotein (LDL) cholesterol (LDL-C) of the premature CAD group was significantly higher than for the propensity score matched later CAD group $(2.50 \pm 0.96$ vs. $2.17 \pm 0.80 \mathrm{mmol} / \mathrm{L}$, $\mathrm{p}=0.019)$. Renal function was significantly better in the premature $\mathrm{CAD}$ group when compared with the later $\mathrm{CAD}$ group $\left(76.58 \pm 15.96\right.$ vs. $68.80 \pm 14.02 \mathrm{~mL} / \mathrm{min} / 1.73 \mathrm{~m}^{2}$, $\mathrm{p}<0.001$ ) (Table 1). Obviously, eGFR is closely related

Table 1 Baseline clinical characteristics

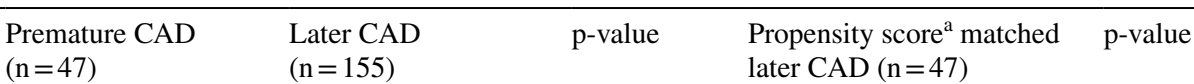

\begin{tabular}{|c|c|c|c|c|c|}
\hline \multicolumn{6}{|l|}{ Patient characteristics } \\
\hline Age, years & $53.53 \pm 7.24$ & $70.48 \pm 8.74$ & $<0.001$ & $72.72 \pm 8.55$ & $<0.001$ \\
\hline Male gender & $24(51.06 \%)$ & $110(70.97 \%)$ & 0.014 & $18(38.30 \%)$ & 0.530 \\
\hline Body mass index, $\mathrm{kg} / \mathrm{m}^{2}$ & $23.87 \pm 3.13$ & $23.55 \pm 3.18$ & 0.545 & $24.20 \pm 3.41$ & 0.348 \\
\hline Current smokers & $16(34.04 \%)$ & $67(43.23 \%)$ & 0.262 & $13(27.66 \%)$ & 0.652 \\
\hline Hypertension & $29(61.70 \%)$ & $107(69.03 \%)$ & 0.348 & $32(68.09 \%)$ & 0.517 \\
\hline Diabetes mellitus & $14(29.79 \%)$ & $39(25.16 \%)$ & 0.528 & $11(23.40 \%)$ & 0.804 \\
\hline Heart failure & $8(17.02 \%)$ & $45(29.03 \%)$ & 0.198 & $15(31.91 \%)$ & 0.190 \\
\hline Premature CAD family history & $8(17.02 \%)$ & $22(14.19 \%)$ & 0.683 & $3(6.38 \%)$ & 0.153 \\
\hline Statin use & $10(21.28 \%)$ & $52(33.55 \%)$ & 0.232 & $14(29.79 \%)$ & 0.466 \\
\hline Homocysteine, $\mu \mathrm{mol} / \mathrm{L}$ & $18.60 \pm 5.15$ & $17.08 \pm 4.27$ & 0.043 & $18.13 \pm 5.74$ & 0.677 \\
\hline Acute coronary syndrome, & $26(55.32 \%)$ & $97(62.58 \%)$ & 0.372 & $31(65.96 \%)$ & 0.826 \\
\hline eGFR-CKD-EPI, mL/min/1.73 m² & $76.58 \pm 15.96$ & $73.16 \pm 15.49$ & 0.189 & $68.80 \pm 14.02$ & $<0.001$ \\
\hline HbA1c, $\%$ & $5.60(5.30-6.60)$ & $5.70(5.30-7.00)$ & 0.846 & $5.80(5.40-7.00)$ & 0.477 \\
\hline \multicolumn{6}{|l|}{ Lipid profile at baseline, $\mathrm{mmol} / \mathrm{L}$} \\
\hline Total cholesterol & $4.19 \pm 1.18$ & $3.89 \pm 1.08$ & 0.107 & $4.01 \pm 0.89$ & 0.424 \\
\hline HDL cholesterol & $1.08 \pm 0.29$ & $1.06 \pm 0.54$ & 0.807 & $0.99 \pm 0.21$ & 0.838 \\
\hline LDL cholesterol & $2.50 \pm 0.96$ & $2.24 \pm 0.88$ & 0.084 & $2.17 \pm 0.80$ & 0.019 \\
\hline Triglyceride & $1.83 \pm 1.08$ & $1.55 \pm 0.84$ & 0.072 & $1.82 \pm 0.96$ & 0.759 \\
\hline C-reactive protein, $\mathrm{mg} / \mathrm{L}$ & $4.00(3.00-6.00)$ & $3.00(3.00-5.00)$ & 0.386 & $4.00(3.00-5.00)$ & 0.150 \\
\hline
\end{tabular}

Data are expressed as mean \pm standard deviation or median (interquartile range) or number (\%)

$C A D$ coronary artery disease, $e G F R$ estimated glomerular filtration rate, $C K D$ chronic kidney disease, $E P I$ exocrine pancreatic insufficiency, $H b A l c$ glycated hemoglobin, $H D L$ high-density lipoprotein, $L D L$ low-density lipoprotein, $B M I$ body mass index, $A C S$ acute coronary syndrome

${ }^{a}$ Propensity score was matched by gender, BMI, indication for catheterization (ACS or stable CAD), hypertension, diabetes mellitus, statin use, and current smoking 
with age, a comparison between same age groups would be more suitable.

\section{Plaque characteristics from coronary angiography}

The plaque characteristics as classified by coronary angiography are summarized in Table 2 . For the whole patient population, the target lesions occurred most often in the left anterior descending artery. It seems that proportion of complex lesions in later CAD group was higher than that in premature CAD group, but the difference was not significant after the matching procedure (Table 2).

\section{Plaque characteristics from iMap-IVUS}

The iMap-IVUS data are listed in Table 3. Before the matching procedure, the target lesion length in the premature CAD group was shorter than that in the later CAD group [18.50 (12.60-32.00) vs. $27.90(18.70-37.40) \mathrm{mm}$, $\mathrm{p}=0.002]$, besides, the plaque volume was much less in the premature CAD group [175.59 (96.60-240.50)] vs. 214.73 (139.74-330.00) $\left.\mathrm{mm}^{3}, \mathrm{p}=0.013\right]$, although these differences were not significant after the matching procedure. Furthermore, in the premature CAD group, the plaque burden was lower compared to the later CAD group $(72.69 \pm 9.99$ vs. $74.85 \pm 9.80 \%, \mathrm{p}=0.005)$ with less negative remodeling $(1.03 \pm 0.12$ vs. $0.94 \pm 0.18, p=0.034)$. Overall, the proportion of each of these 4 plaque components was significantly different between the premature and later CAD groups. At the minimum lumen CSA site in each plaque, the premature CAD appears to have more fibrotic area $(66.47 \pm 16.26$ vs. $53.06 \pm 19.45, \mathrm{p}<0.001)$, less necrotic area $(21.28 \pm 12.51$ vs. $27.70 \pm 15.84 \%, \mathrm{p}=0.032)$ and significantly less calcified area $[0.04(0.02-0.12)$ vs. $1.56(0.42-3.49), p=0.012]$ than in the later $\mathrm{CAD}$ group. However, the difference in plaque components for the whole plaque was not significant (Fig. 1). Besides, the incidence of high risk features: plaque burden of $\geq 70 \%$, and iMap IVUS derived thin-cap fibroatheroma (TCFA), were significantly lower in premature CAD group (72.34 vs. $89.36 \%, p=0.036,59.57$ vs. $85.11 \%, p=0.006$ respectively) (Table 3 ).

\section{Discussion}

The aim of this study was to characterize coronary plaque tissue by iMap-IVUS in patients with premature CAD and compare the results with older patients with CAD. The main findings of the study were as follows: in the premature $\mathrm{CAD}$ group compared with the later CAD group, (1) more fibrotic and less necrotic and calcified components were observed. (2) High-risk plaques in terms of plaque burden $\geq 70 \%$ and TCFA were observed less in the target lesion. (3) The range and degree of atherosclerosis appears to be lower (number of diseased vessels, lesion length, plaque volume) (Fig. 2).

Previous studies that focused on gender differences showed similar results by Virtual Histology and grey-scale IVUS [18-21]. However, these studies did not match for baseline factors in different aged groups, and these factors may bias the results. Therefore, we focused on plaque characteristics in premature CAD compared with later CAD using propensity score matching for gender, BMI and a few other cardiovascular risk factors. We also controlled the time of onset to within three months to reduce the interference from different disease duration as much as possible. Contrary to these results, a large study found that younger patients had more unstable plaque morphology [21], the possible reasons for the different result are that we excluded patients with visible thrombus and TCFA could not predict prognosis precisely within these limits [12].

Previous grey-scale IVUS studies have demonstrated that elderly patients had more calcified plaques, more negative remodeling and diffuse atherosclerosis [21]. With age vessels become dilated, tortuous, calcified, and

Table 2 Plaque characteristics from coronary angiography

\begin{tabular}{lcccc}
\hline & $\begin{array}{l}\text { Premature CAD } \\
(\mathrm{n}=47)\end{array}$ & $\begin{array}{l}\text { Later CAD } \\
(\mathrm{n}=155)\end{array}$ & $\mathrm{p}$-value & $\begin{array}{l}\text { Propensity score matched } \\
\text { later CHD (n=47) }\end{array}$ \\
\hline Target artery & & & 0.870 & $25(53.19 \%)$ \\
LAD & $29(61.70 \%)$ & $92(59.35 \%)$ & & $12(25.53 \%)$ \\
RCA & $10(21.28 \%)$ & $36(23.23 \%)$ & & $10(21.28 \%)$ \\
LCX & $8(17.02 \%)$ & $27(17.42 \%)$ & & $11(23.40 \%)$ \\
Complex lesion B2C & $9(19.1 \%)$ & $55(35.5 \%)$ & 0.035 & 0.705 \\
Number of diseased vessels & $1.00(1.00-2.00)$ & $1.00(1.00-2.00)$ & 0.762 & $2.00(1.00-3.00)$ \\
\hline
\end{tabular}

Data are expressed as median (interquartile range) or number (\%)

$C A D$ coronary artery disease, $L A D$ left anterior descending, $R C A$ right coronary artery, $L C X$ left circumflex

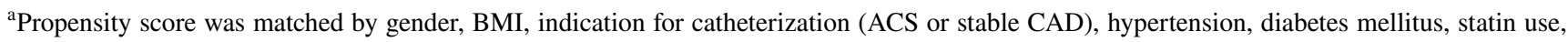
and current smoking 
Table 3 iMap-IVUS segment and lesion characteristics in the target artery

\begin{tabular}{|c|c|c|c|c|c|}
\hline & $\begin{array}{l}\text { Premature CAD } \\
(\mathrm{n}=47)\end{array}$ & $\begin{array}{l}\text { Later CAD } \\
(\mathrm{n}=155)\end{array}$ & p-value & $\begin{array}{l}\text { Propensity score }{ }^{a} \text { matched } \\
\text { Later CHD }(n=47)\end{array}$ & p-value \\
\hline \multicolumn{6}{|l|}{ iMap IVUS analysis (2D) } \\
\hline Lumen $\mathrm{CSA}^{\mathrm{b}}, \mathrm{mm}^{2}$ & $3.63 \pm 1.49$ & $3.36 \pm 1.99$ & 0.404 & $3.12 \pm 1.89$ & 0.154 \\
\hline $\mathrm{EEM} \mathrm{CSA}^{\mathrm{b}}, \mathrm{mm}^{2}$ & $12.78(10.37-17.20)$ & $13.96(11.13-16.41)$ & 0.467 & $13.10(11.01-15.81)$ & 0.719 \\
\hline Plaque $\mathrm{CSA}^{\mathrm{b}}, \mathrm{mm}^{2}$ & $10.23 \pm 4.30$ & $10.89 \pm 4.08$ & 0.342 & $11.12 \pm 4.25$ & 0.319 \\
\hline Plaque burden, $\%$ & $72.69 \pm 9.99$ & $75.97 \pm 10.46$ & 0.059 & $74.85 \pm 9.80$ & 0.005 \\
\hline Remodeling index & $1.03 \pm 0.12$ & $0.95 \pm 0.24$ & 0.029 & $0.94 \pm 0.18$ & 0.034 \\
\hline Fibrotic area, $\%$ & $66.47 \pm 16.26$ & $51.68 \pm 19.97$ & $<0.001$ & $53.06 \pm 19.45$ & $<0.001$ \\
\hline Lipidic area, $\%$ & $11.49 \pm 4.58$ & $12.54 \pm 4.76$ & 0.185 & $12.98 \pm 4.89$ & 0.131 \\
\hline Necrotic area, $\%$ & $18.32(10.05-30.93)$ & $30.25(20.24-49.33)$ & $<0.001$ & $30.84(20.04-47.33)$ & 0.001 \\
\hline Calcified area, $\%$ & $0.43(0.19-1.22)$ & $1.26(0.38-3.14)$ & 0.002 & $1.56(0.42-3.49)$ & 0.012 \\
\hline Fibrotic area, $\mathrm{mm}^{2}$ & $6.61 \pm 2.98$ & $5.33 \pm 2.39$ & 0.003 & $5.60 \pm 2.50$ & 0.080 \\
\hline Lipidic area, $\mathrm{mm}^{2}$ & $1.20 \pm 0.77$ & $1.42 \pm 0.88$ & 0.119 & $1.50 \pm 0.89$ & 0.079 \\
\hline Necrotic area, $\mathrm{mm}^{2}$ & $1.79(0.89-4.14)$ & $4.00(1.46-7.02)$ & 0.002 & $3.45(1.54-5.52)$ & 0.003 \\
\hline Calcified area, $\mathrm{mm}^{2}$ & $0.04(0.02-0.12)$ & $0.12(0.04-0.29)$ & $<0.001$ & $0.10(0.05-0.25)$ & 0.008 \\
\hline \multicolumn{6}{|l|}{ iMap-IVUS analysis (3D) } \\
\hline Lumen volume, $\mathrm{mm}^{3}$ & $87.75(52.62-156.60)$ & $140.07(79.17-225.96)$ & 0.005 & $143.92(81.92-209.08)$ & 0.036 \\
\hline Vessel volume, $\mathrm{mm}^{3}$ & 238.85 (153.04-389.04) & $376.92(234.80-564.52)$ & 0.004 & 356.94 (178.36-504.90) & 0.080 \\
\hline Plaque volume, $\mathrm{mm}^{3}$ & $175.59(96.60-240.50)$ & $214.73(139.74-330.00)$ & 0.013 & $204.21(106.70-311.13)$ & 0.182 \\
\hline PAV, $\%$ & $62.72 \pm 13.11$ & $60.28 \pm 11.64$ & 0.222 & $61.12 \pm 11.59$ & 0.569 \\
\hline Fibrotic volume, $\%$ & $61.37 \pm 14.50$ & $57.90 \pm 13.76$ & 0.136 & $59.00 \pm 11.85$ & 0.388 \\
\hline Lipidic volume, $\%$ & $12.00 \pm 3.54$ & $12.65 \pm 2.96$ & 0.210 & $12.37 \pm 2.09$ & 0.539 \\
\hline Necrotic volume, $\%$ & $24.74 \pm 10.98$ & $27.32 \pm 11.34$ & 0.173 & $26.21 \pm 9.74$ & 0.494 \\
\hline Calcified volume, $\%$ & $2.76(1.00-4.14)$ & $3.23(1.26-6.21)$ & 0.165 & $2.30(1.14-4.73)$ & 0.284 \\
\hline Fibrotic volume, $\mathrm{mm}^{3}$ & $95.89(52.50-163.43)$ & $122.57(74.53-188.65)$ & 0.059 & $125.31(65.08-163.55)$ & 0.243 \\
\hline Lipidic volume, $\mathrm{mm}^{3}$ & $16.89(11.97-31.20)$ & $24.32(15.02-42.00)$ & 0.018 & $23.14(12.80-34.98)$ & 0.320 \\
\hline Necrotic volume, $\mathrm{mm}^{3}$ & $29.64(21.21-69.60)$ & $51.87(28.73-103.12)$ & 0.004 & $48.52(25.68-71.89)$ & 0.163 \\
\hline Calcified volume, $\mathrm{mm}^{3}$ & $4.00(1.78-7.20)$ & $5.60(2.62-13.27)$ & 0.031 & $4.02(2.06-10.04)$ & 0.535 \\
\hline \multicolumn{6}{|c|}{ High risk lesion characteristics } \\
\hline Plaque burden $\geq 70 \%$ & $34(72.34 \%)$ & $125(80.65 \%)$ & 0.223 & $42(89.36 \%)$ & 0.036 \\
\hline MLA $\leq 4.0 \mathrm{~mm}^{2}$ & $33(70.21 \%)$ & $117(75.48 \%)$ & 0.469 & $40(85.11 \%)$ & 0.083 \\
\hline TCFA & $28(59.57 \%)$ & $122(78.71 \%)$ & 0.009 & $40(85.11 \%)$ & 0.006 \\
\hline
\end{tabular}

Data are expressed as mean \pm standard deviation or median (interquartile range) or number (\%)

$C A D$ coronary artery disease, IVUS intravascular ultrasound, $B M I$ body mass index, $A C S$ acute coronary syndrome, $M L A$ minimal lumen area, TCFA thin-cap fibroatheroma, PAV percent atheroma volume, EEM external elastic membrane, CSA cross sectional area

${ }^{a}$ Propensity score was matched by gender, BMI, indication for catheterization (ACS or stable CAD), hypertension, diabetes mellitus, statin use, and current smoking

${ }^{b}$ Measuring at the minimum lumen CSA site in each plaque would been properly for comparion

dysfunctional, according to classical pathologic and animal model experiments [22, 23]. Young female patients with CAD are rarer than males, and the degree of atherosclerosis was lower in female premature CAD patients. With increasing age, the difference between genders becomes less [18-20]. On the other hand, the plaques of young female patients showed more cellular fibrous tissue and lipid-rich foam cells, which reveals the reversibility of CAD in younger patients [24].
Traditional cardiovascular risk factors are closely associated with premature CAD. Patients with premature myocardial infarction appear to have a higher prevalence of smoking, family history of premature CAD and male gender [25]. Controversially, the degree of hyperlipidemia, hypertension and diabetes does not appear to be as robust as the risk factors already discussed [25]. In this study, there was no significant difference in traditional risk factors between the groups except homocysteine, but after propensity score 


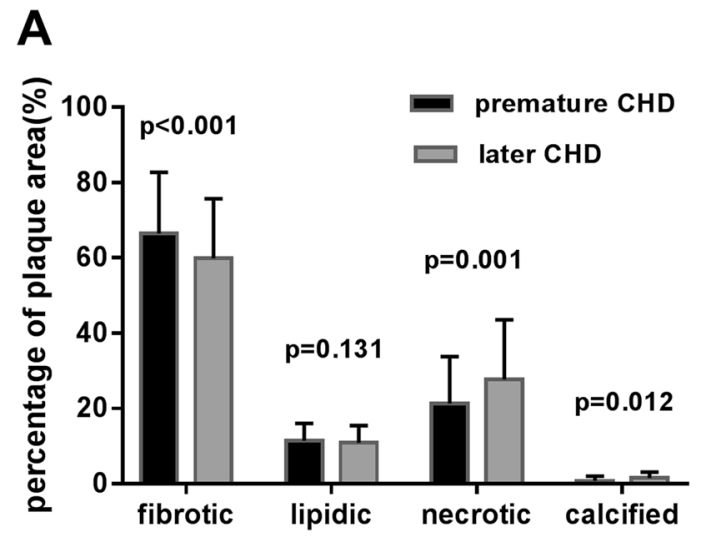

B
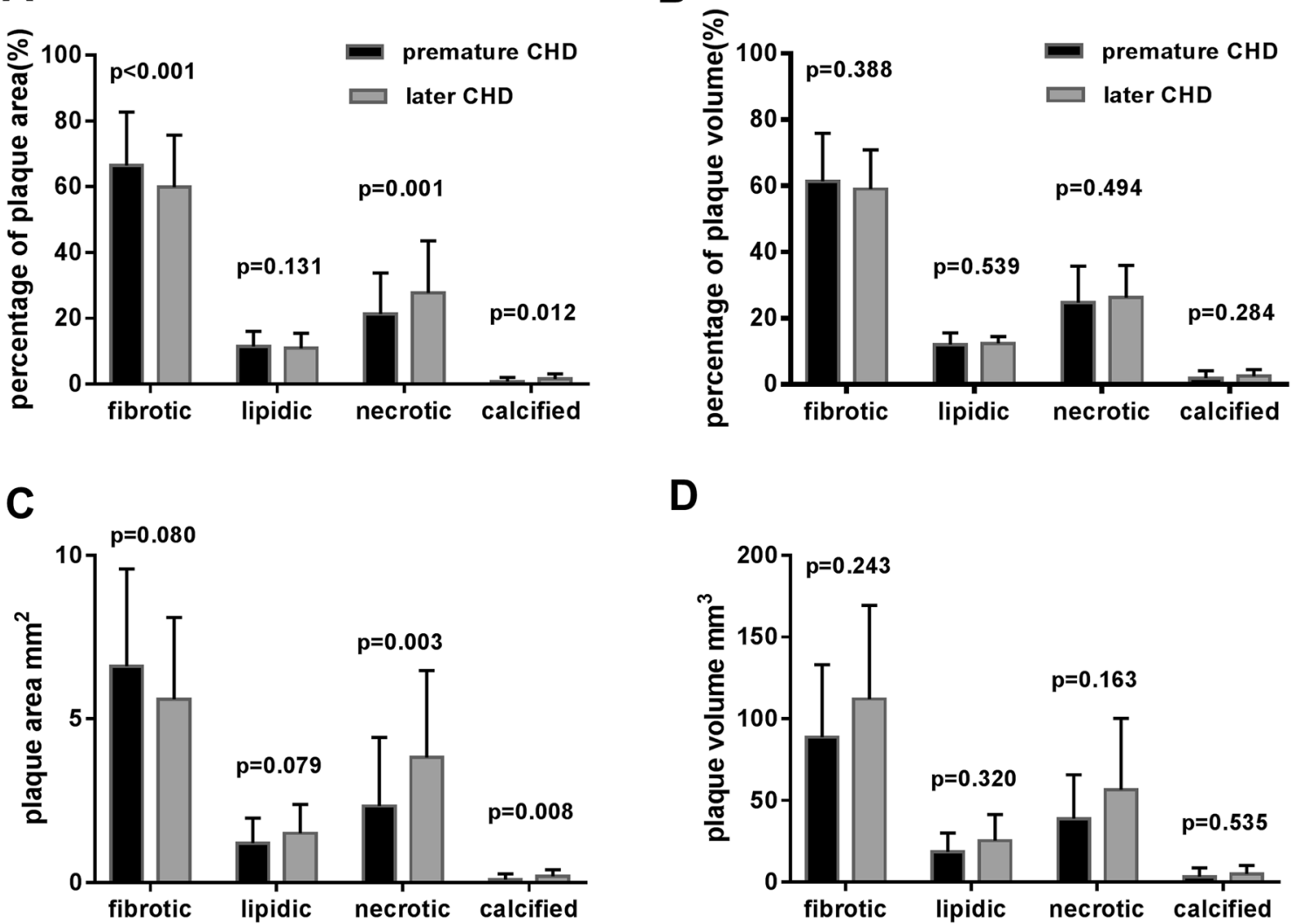

D

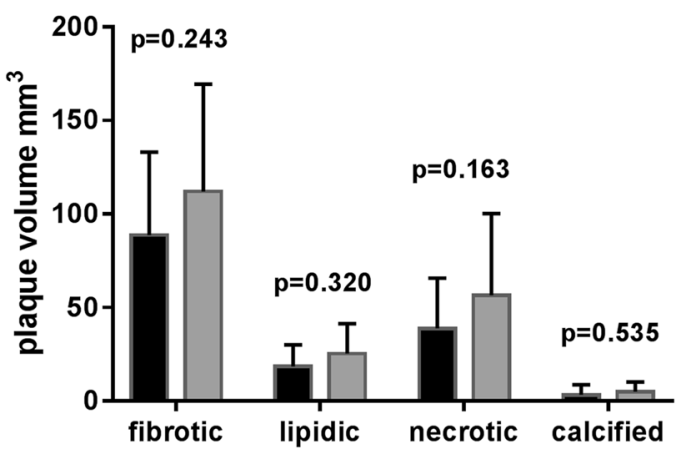

Fig. 1 At the minimum lumen cross sectional area (CSA) site in each plaque, the percent fibrotic was higher, percent necrotic and calcified was lower in premature CAD group than later CAD (a). Between the two groups, there were significant differences in necrotic and calcified

areas (b). In the whole plaque, the difference of plaque components between the two groups was not significant $(\mathbf{c})$. The mean plaque volume in the later CAD group was greater than that in the premature CAD group, and every component was greater too $(\mathbf{d})$

matching only LDL-C was significantly higher in the premature CAD group. Dyslipidemia plays an important role in development of atherosclerosis, which has been explored a lot in the field of genetics. Compared with the general population, hyperlipidemia is related to autosomal genetic mutations which are characterized by severe elevations in LDL-C which could increase a concomitant 10-20 fold risk of premature CAD [26]. Among these genetic factors, LDLreceptor mutations are the most common genetic defect in all individuals with premature CAD [27]. In the future, proprotein convertase subtilisin-kexin type 9 (PCSK9) inhibitors are expected to ameliorate hyperlipidemia due to genetic causes [28]. For now, statins are effective and economical lipid-lowering therapies. Importantly, these studies suggest that patients with premature CAD could have more benefit from statin therapy than elderly patients [29].

In the general population, serum homocysteine level is associated with age and renal function [30]. However, in this study, premature CAD patients with better renal function and younger age showed a higher level of homocysteine. It should be considered that heredity and metabolize factors

are involved in premature CAD [31]. Compared with nonCAD individuals, the level of homocysteine is remarkably higher in patients with CAD especially younger patients, and this has been regarded as an independent risk factor for arteriosclerosis in southern China crowd [32]. Hyperhomocyteinemia is involved in arteriosclerosis through several mechanisms, such as endothelial dysfunction, permeability of cholesterol and inflammatory cells, vascular inflammation, lipoprotein oxidation, smooth muscle proliferation, platelet activation, and abnormalities in the clotting cascade [31, 33, 34]. However, there is much controversy still exists regarding homocysteine as a cardiovascular risk factor. Common genetic variants that influence homocysteine level are not related with risk of CAD in white populations [35]. In fact, most clinical trials focus on supplementation of folic acid with or without vitamin B to ameliorate hyperhomocysteinemia do not reduce the relative risk for CAD [36].

Although this study did not focus on smoking cessation, it seems to be the most important risk factor modification for premature CAD. Current smokers appear to have more lipid-rich plaques than those who had never 
Fig. 2 Example of premature CAD plaque characterization at CSA using gray-scale IVUS and iMap-IVUS and compared to the later CAD. a A-55-year-old female plaque was composed of fibrotic area $(90.89 \%)$, lipidic area $(2.81 \%)$, necrotic area $(5.68 \%)$ and calcified area $(0.62 \%)$. b A-70-yeas-old male plaque was composed of fibrotic area (66.26\%), lipidic area $(12.64 \%)$, necrotic area $(17.72 \%)$ and calcified area $(3.38 \%)$

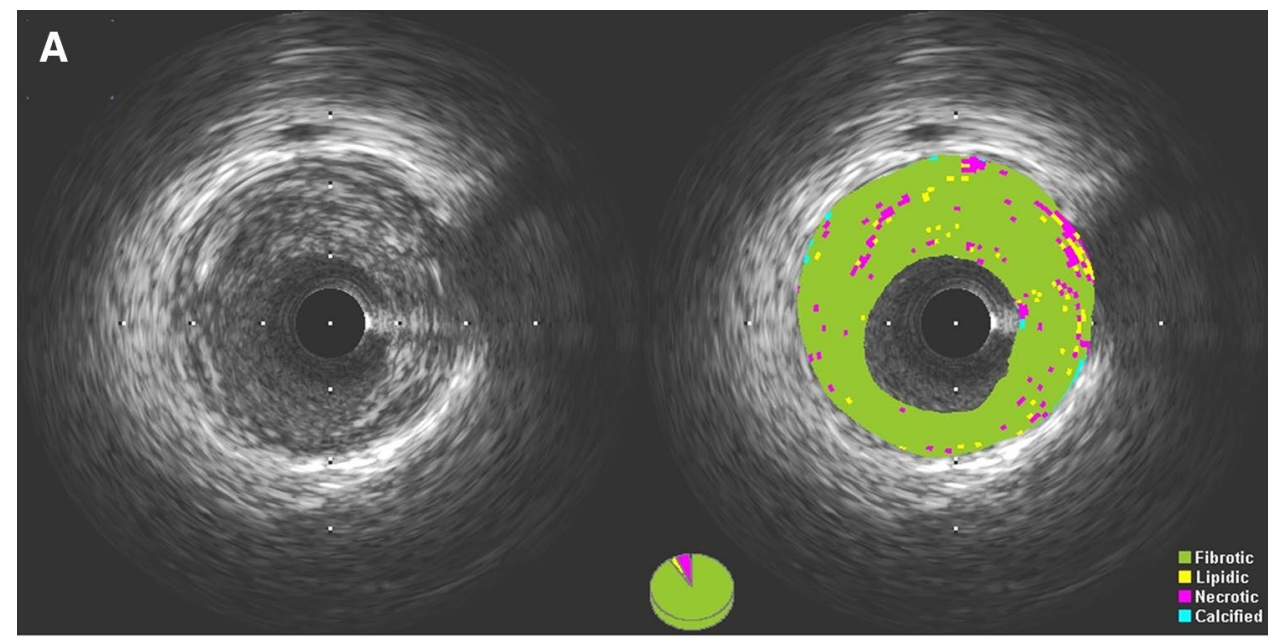

B

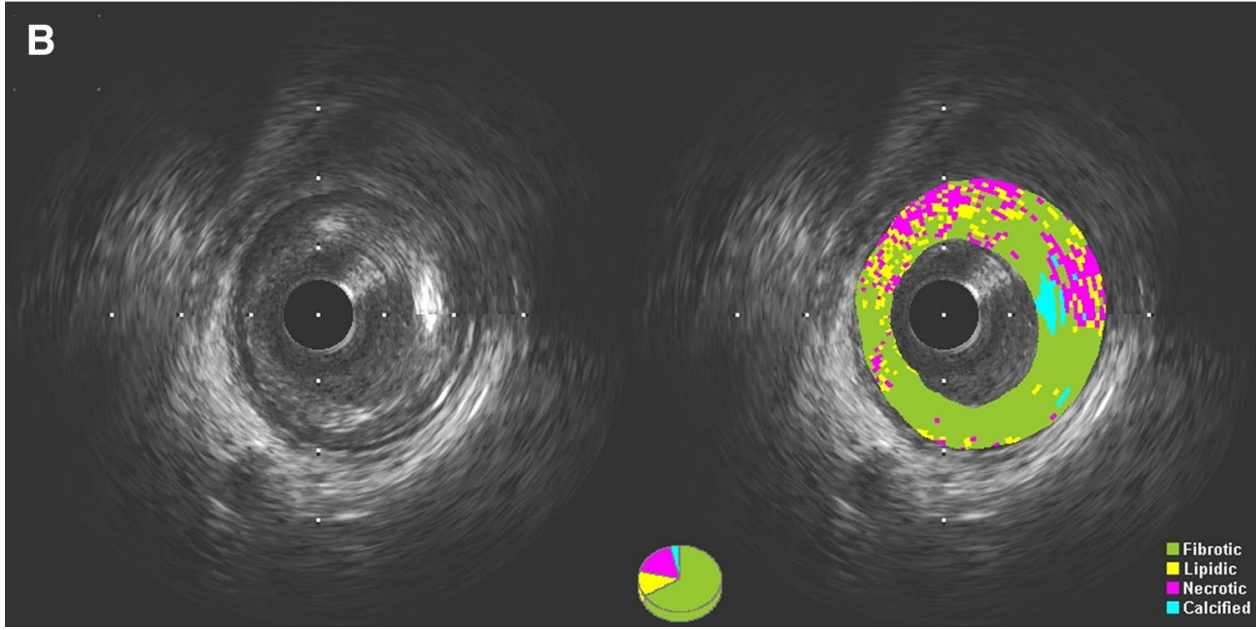

smoked, or quit more than 1 year ago [37, 38]. After MI, continued smokers with a relative risk of 1.51 (95\% CI 1.10-2.07) recurrent coronary events compared with nonsmokers, while the risk declined to equal that of nonsmokers 3 years after cessation [39].

This study has some limitations. This was a retrospective study performed at a single center with a relatively small sample size, so selection bias cannot be excluded. In order to avoid the misrecognition of iMap-IVUS to thrombus, some patients with STEMI were excluded. Acoustic shadowing behind calcification or wire artefact makes the analysis incomplete, and these "unknown areas" can often be mistaken for necrotic areas. Different from stable CAD, patients with ACS appears to have less fibrotic, more necrotic and lipidic component [40]. After matching procedure, a higher proportion of patients with ACS in later CAD may affect results. In small sample sizes, propensity match analyses could not exclude some potential cofounders. More samples are required for subgroup analysis in ACS group in future.

\section{Conclusions}

Coronary plaque tissue in premature CAD appears to be more fibrotic with less necrotic and calcified components compared with later CAD, the range and degree of atherosclerosis were significantly lower than in later CAD.

Acknowledgements This work was supported by a Grant-in-Aid for Clinical Scientific Research Project from institute of medicine of Zhejiang (2017ZYC-A38); Zhejiang Province Nature Science Foundation (Y15H020003); Hangzhou Health Science and technology project (201884436); Zhejiang Province Science and Technology Public Welfare Project (2016C33SA100056).

\section{Compliance with ethical standards}

Conflict of interest All authors declare that they have no confict of interest.

Ethical approval The study protocol conformed to the ethical guidelines of the Declaration of Helsinki. 
Open Access This article is licensed under a Creative Commons Attribution 4.0 International License, which permits use, sharing, adaptation, distribution and reproduction in any medium or format, as long as you give appropriate credit to the original author(s) and the source, provide a link to the Creative Commons licence, and indicate if changes were made. The images or other third party material in this article are included in the article's Creative Commons licence, unless indicated otherwise in a credit line to the material. If material is not included in the article's Creative Commons licence and your intended use is not permitted by statutory regulation or exceeds the permitted use, you will need to obtain permission directly from the copyright holder. To view a copy of this licence, visit http://creativecommons.org/licenses/by/4.0/.

\section{References}

1. Nichols M, Townsend N, Scarborough P, Rayner M (2014) Cardiovascular disease in Europe 2014: epidemiological update. Eur Heart J 35(42):2950-2959

2. Camargo GC, Rothstein T, Derenne ME et al (2017) Factors associated with coronary artery disease progression assessed by serial coronary computed tomography angiography. Arq Bras Cardiol 108(5):396-404

3. Shah M, Sikkel MB (2013) Coronary artery disease and age: beyond atherosclerosis. J Physiol 591(23):5807-5808

4. Abderrahman HA, Al-Abdallat IM, Idhair AK (2018) Age threshold for proper definition of premature coronary artery disease in males. J Forensic Leg Med 58:45-49

5. Wang X, Gao M, Zhou S et al (2017) Trend in young coronary artery disease in China from 2010 to 2014: a retrospective study of young patients $\leq 45$. BMC Cardiovasc Disord 17(1):18

6. Joshi P, Islam S, Pais P et al (2007) Risk factors for early myocardial infarction in South Asians compared with individuals in other countries. JAMA 297(3):286-294

7. Panwar RB, Gupta R, Gupta BK et al (2011) Atherothrombotic risk factors \& premature coronary heart disease in India: a casecontrol study. Indian J Med Res 134:26-32

8. Nasir K, Budoff MJ, Wong ND et al (2007) Family history of premature coronary heart disease and coronary artery calcification: multi-ethnic study of atherosclerosis (MESA). Circulation 116(6):619-626

9. Christiansen MK, Jensen JM, Nørgaard BL, Dey D, Bøtker HE, Jensen HK (2017) Coronary plaque burden and adverse plaque characteristics are increased in healthy relatives of patients with early onset coronary artery disease. JACC 10(10):1128-1135

10. Ochijewicz D, Tomaniak M, Koltowski L, Rdzanek A, Pietrasik A, Kochman J (2017) Intravascular imaging of coronary artery disease: recent progress and future directions. J Cardiovasc Med (Hagerstown) 18(10):733-741

11. Higuchi $Y$, Hiro T, Takayama $T$ et al (2014) Impact of coronary plaque burden and composition on periprocedural myocardial infarction and coronary flow reserve after percutaneous coronary intervention. Int Heart J 55(5):391-396

12. Stone GW, Maehara A, Lansky AJ et al (2011) A prospective natural-history study of coronary atherosclerosis. N Engl J Med 364(3):226-235

13. Ma T, Zhou B, Hsiai TK, Shung KK (2016) A review of intravascular ultrasound-based multimodal intravascular imaging: the synergistic approach to characterizing vulnerable plaques. Ultrason Imaging 38(5):314-331

14. Sathyanarayana S, Carlier S, Li W, Thomas L (2009) Characterisation of atherosclerotic plaque by spectral similarity of radiofrequency intravascular ultrasound signals. Eurointervention $5(1): 133-139$
15. Katouzian A, Sathyanarayana S, Baseri B, Konofagou EE, Carlier SG (2008) Challenges in atherosclerotic plaque characterization with intravascular ultrasound (IVUS): from data collection to classification. IEEE Trans Inf Technol Biomed 12(3):315-327

16. Mintz GS, Nissen SE, Anderson WD et al (2001) American College of Cardiology Clinical Expert Consensus Document on Standards for Acquisition, Measurement and Reporting of Intravascular Ultrasound Studies (IVUS). A report of the American College of Cardiology Task Force on Clinical Expert Consensus Documents. J Am Coll Cardiol 37(5):1478-1492

17. Araki T, Nakamura M, Utsunomiya M, Sugi K (2012) Visualization of coronary plaque in type 2 diabetes mellitus patients using a new $40 \mathrm{MHz}$ intravascular ultrasound imaging system. J Cardiol 59(1):42-49

18. Ann SH, De Jin C, Singh GB et al (2016) Gender differences in plaque characteristics of culprit lesions in patients with ST elevation myocardial infarction. Heart Vessels 31(11):1767-1775

19. Qian J, Maehara A, Mintz GS et al (2009) Impact of gender and age on in vivo virtual histology-intravascular ultrasound imaging plaque characterization (from the global Virtual Histology Intravascular Ultrasound [VH-IVUS] registry). Am J Cardiol 103(9):1210-1214

20. Ruiz-Garcia J, Lerman A, Weisz G et al (2012) Age- and gender-related changes in plaque composition in patients with acute coronary syndrome: the PROSPECT study. Eurointervention 8(8):929-938

21. Hong YJ, Jeong MH, Ahn Y et al (2008) Age-related differences in intravascular ultrasound findings in 1,009 coronary artery disease patients. Circ J 72(8):1270-1275

22. Burke AP, Weber DK, Kolodgie FD, Farb A, Taylor AJ, Virmani R (2001) Pathophysiology of calcium deposition in coronary arteries. Herz 26(4):239-244

23. Lee TJ, Shirasaki Y, Nickols GA (1987) Altered endothelial modulation of vascular tone in aging and hypertension. Blood Vessels 24(3):132-136

24. Dollar AL, Kragel AH, Fernicola DJ, Waclawiw MA, Roberts WC (1991) Composition of atherosclerotic plaques in coronary arteries in women less than 40 years of age with fatal coronary artery disease and implications for plaque reversibility. Am J Cardiol 67(15):1223-1227

25. Shah N, Kelly AM, Cox N, Wong C, Soon K (2016) Myocardial infarction in the "young": risk factors, presentation, management and prognosis. Heart Lung Circ 25(10):955-960

26. Nordestgaard BG, Chapman MJ, Humphries SE et al (2013) Familial hypercholesterolaemia is underdiagnosed and undertreated in the general population: guidance for clinicians to prevent coronary heart disease: consensus statement of the European Atherosclerosis Society. Eur Heart J 34(45):3478-3490

27. Do R, Stitziel NO, Won $\mathrm{HH}$ et al (2015) Exome sequencing identifies rare LDLR and APOA5 alleles conferring risk for myocardial infarction. Nature 518(7537):102-106

28. Lloyd-Jones DM, Morris PB, Ballantyne CM et al (2017) 2017 Focused update of the 2016 ACC expert consensus decision pathway on the role of non-statin therapies for LDL-cholesterol lowering in the management of atherosclerotic cardiovascular disease risk: a report of the american college of cardiology task force on expert consensus decision pathways. J Am Coll Cardiol 70(14):1785-1822

29. Nozue T, Yamamoto S, Tohyama $S$ et al (2014) Impacts of age on coronary atherosclerosis and vascular response to statin therapy. Heart Vessels 29(4):456-463

30. Long Y, Nie J (2016) Homocysteine in renal injury. Kidney Dis (Basel) 2(2):80-87

31. Antoniades C, Antonopoulos AS, Tousoulis D, Marinou K, Stefanadis C (2009) Homocysteine and coronary atherosclerosis: 
from folate fortification to the recent clinical trials. Eur Heart $\mathbf{J}$ 30(1):6-15

32. Wu Y, Huang Y, Hu Y et al (2013) Hyperhomocysteinemia is an independent risk factor in young patients with coronary artery disease in southern China. Herz 38(7):779-784

33. Di Minno MN, Tremoli E, Coppola A, Lupoli R, Di Minno G (2010) Homocysteine and arterial thrombosis: challenge and opportunity. Thromb Haemost 103(5):942-961

34. Banos-Gonzalez MA, Angles-Cano E, Cardoso-Saldana G et al (2012) Lipoprotein(a) and homocysteine potentiate the risk of coronary artery disease in male subjects. Circ J 76(8):1953-1957

35. Bazzano LA, Reynolds K, Holder KN, He J (2006) Effect of folic acid supplementation on risk of cardiovascular diseases: a meta-analysis of randomized controlled trials. JAMA 296(22):2720-2726

36. van Meurs JB, Pare G, Schwartz SM et al (2013) Common genetic loci influencing plasma homocysteine concentrations and their effect on risk of coronary artery disease. Am J Clin Nutr 98(3):668-676

37. Buljubasic N, Akkerhuis KM, de Boer SP et al (2015) Smoking in relation to coronary atherosclerotic plaque burden, volume and composition on intravascular ultrasound. PLoS ONE 10(10):e141093

38. Kumagai S, Amano T, Takashima H et al (2015) Impact of cigarette smoking on coronary plaque composition. Coron Artery Dis 26(1):60-65

39. Rea TD, Heckbert SR, Kaplan RC, Smith NL, Lemaitre RN, Psaty BM (2002) Smoking status and risk for recurrent coronary events after myocardial infarction. Ann Intern Med 137(6):494-500

40. Kozuki A, Shinke T, Otake H et al (2013) Feasibility of a novel radiofrequency signal analysis for in-vivo plaque characterization in humans: comparison of plaque components between patients with and without acute coronary syndrome. Int J Cardiol 167(4):1591-1596 